\title{
Knockdown of p66Shc Alters Lineage-Associated Transcription Factor Expression in Mouse Blastocysts
}

\author{
Nicole A. Edwards, Andrew J. Watson, ${ }^{1-3}$ and Dean H. Betts ${ }^{1-3}$
}

\begin{abstract}
The p66Shc adaptor protein regulates apoptosis and senescence during early mammalian development. However, p66Shc expression during mouse preimplantation development is upregulated at the blastocyst stage. Our objective was to determine the biological function of p66Shc during mouse blastocyst development. In this study, we demonstrate that a reduced p66Shc transcript abundance following its short interfering RNA (siRNA)-mediated knockdown alters the spatiotemporal expression of cell lineage-associated transcription factors in the inner cell mass (ICM) of the mouse blastocyst. P66Shc knockdown blastocysts restrict OCT3/4 earlier to the inner cells of the early blastocyst and have ICMs containing significantly higher OCT3/4 levels, more GATA4-positive cells, and fewer NANOG-positive cells. P66Shc knockdown blastocysts also show a significantly reduced ability to form ICMderived outgrowths when explanted in vitro. The increase in cells expressing primitive endoderm markers may be due to increased ERK1/2 activity, as it is reversed by ERK1/2 inhibition. These results suggest that p66Shc may regulate the relative abundance and timing of lineage-associated transcription factor expression in the blastocyst ICM.
\end{abstract}

Keywords: p66Shc, blastocyst, epiblast, inner cell mass, MAPK, primitive endoderm

\section{Introduction}

$\mathrm{M}$ OUSE PREIMPLANTATION EMBRYO development results in the formation of a blastocyst containing three distinct cell types: the trophectoderm (TE), the primitive endoderm (PE), and the epiblast (EPI). Postimplantation, the TE and PE form extraembryonic structures, whereas the EPI will contribute to the fetal germ layers and germ cells. Proper specification of these three cell lineages requires spatiotemporal activation of signaling pathways that promote the expression of lineage-specific markers, some of which are required to maintain cell fate and identity.

Studies show that in the 8-16 cell embryo, the Hippo signaling pathway is the main signaling pathway critical to establishing TE versus inner cell mass (ICM) cell identity by regulating the expression of the TE-specific marker CDX2 [1-3]. In the blastocyst, differential fibroblast growth factor 4 (FGF4)/ mitogen-activated protein kinase (MAPK) signaling drives the acquisition of an EPI or PE fate in the ICM by regulating the expression of the EPI marker NANOG and the PE markers GATA6, SOX17, and GATA4 [4-7]. Disrupting components of either signaling pathway results in aberrant cell fate specification and failure to maintain embryonic development postimplantation, or failure to generate lineage-specific stem cells $[2-4,8]$.
Of these signaling pathway components, receptor tyrosine kinase (RTK) signaling in response to FGF4 binding is required for $\mathrm{PE}$ cell fate determination. Growth factor receptorbound protein 2 (GRB2) is an adaptor protein linking activated RTKs to Ras/MAPK signaling. Grb2 knockout (KO) embryos have ICMs containing no PE cells as identified by the absence of Gata6 expression. Instead, all cells of Grb2 KO blastocyst ICMs are NANOG positive [9]. These results therefore demonstrate that MAPK signaling downstream of RTK activation is required for expression of PE-specific markers and PE specification.

Similarly, embryos treated with the extracellular signalregulated kinase (ERK) inhibitors from the 8-cell to the blastocyst stage generate ICMs containing all EPI cells [5,7]. However, this phenotype is partially reversible if the inhibitor is removed by embryonic day 3.75 (E3.75), indicating that ICM cells maintain plasticity until E4.0-E4.5 [5]. Similarly, cell aggregation experiments showed that ICM cells lose this plasticity by E4.5 [10]. Thus, MAPK signaling is important for stabilizing PE specification in the blastocyst until commitment occurs just before implantation.

Another RTK signaling pathway component expressed in many cell types is the family of SHC1 adaptor proteins. All Shc1 isoforms contain a common phosphotyrosine-binding domain that associates with activated RTKs, but unlike

\footnotetext{
Departments of ${ }^{1}$ Physiology and Pharmacology, ${ }^{2}$ Obstetrics and Gynecology, Schulich School of Medicine \& Dentistry, The University of Western Ontario, London, Canada.

${ }^{3}$ The Children's Health Research Institute (CHRI), Lawson Health Research Institute, London, Canada.

(C) Nicole A. Edwards et al. 2018; Published by Mary Ann Liebert, Inc. This Open Access article is distributed under the terms of the Creative Commons Attribution Noncommercial License (http://creativecommons.org/licenses/by-nc/4.0/) which permits any noncommercial use, distribution, and reproduction in any medium, provided the original author(s) and the source are cited.
} 
p52Shc, p66Shc does not activate downstream Ras-MAPK signaling $[11,12]$. A unique function of p66Shc is in the response to oxidative stress attributed to serine/threonine sites on an N-terminal extension. Under conditions of oxidative stress, p66Shc is phosphorylated at serine-36, translocates to the mitochondria, and promotes the release of reactive oxygen species (ROS), leading to apoptosis [13]. We have demonstrated that p66Shc is basally expressed in mouse preimplantation embryos, is upregulated at the blastocyst stage, and that its expression is modulated by the culture environment [14]. Loss of function studies using RNA interference (RNAi) showed that p66Shc promotes apoptosis and senescence associated with an increase in ROS in cow and mouse embryos exposed to stress-inducing environmental conditions [1517]. However, whether p66Shc has a biological function that is required to ensure proper preimplantation development, remains unknown.

Due to its role in RTK/MAPK signaling in other cell types, we hypothesized that p66Shc is a regulatory component in the pathways underlying blastocyst cell lineage specification. Thus, the objective was to determine the role of p66Shc in mouse blastocyst development using short interfering RNA (siRNA) knockdown in mouse preimplantation embryos. Our results show that mouse embryos with decreased p66Shc levels formed blastocysts with faster restriction to and higher levels of OCT3/4 in the inner cells, had an earlier onset of GATA4 expression, and earlier sorting of PE cells to the PE layer. P66Shc knockdown ICMs contained significantly more cells expressing PE markers (GATA4, SOX17) than cells expressing EPI markers (NANOG), associated with an increase in cells expressing the ERK1/2 transcriptional target DUSP4. Thus, we have uncovered a novel role for p66Shc associated with the timing and expression of lineage-associated transcription factors in the ICM of mouse blastocysts.

\section{Materials and Methods}

\section{Animal source and ethical approval}

Female and male wild-type CD1 mice were obtained from Charles River Canada (Saint-Constant, Quebec, Canada). Mice were housed with a 12-h light/12-h dark cycle and access to food and water ad libitum. All experimental protocols were approved by the University of Western Ontario Animal Care and Veterinary Services and the Canadian Council of Animal Care (protocol Watson no. 2010-021). For all experiments, mice were euthanized by $\mathrm{CO}_{2}$ asphyxiation.

\section{Mouse zygote collection and culture}

Three- to four-week-old female CD1 mice were superovulated by intraperitoneal (i.p.) injection of pregnant mare serum gonadotropin (Merck Animal Health, Canada) followed by i.p. injection of human chorionic gonadotropin (Merck Animal Health) $48 \mathrm{~h}$ later. Female mice were then singly housed with male mice for mating. The following morning, female mice were checked for the presence of a vaginal plug. Females with vaginal plugs were euthanized and oviducts were dissected. Zygotes were collected by flushing the oviducts with M2 medium (Sigma-Aldrich, Canada). To remove cumulus cells, zygotes were briefly incubated in M2 medium containing hyaluronidase (Zenith Biotech). Zygotes were washed through drops of M2 medium, followed by washes in embryo culture medium (potassium simplex optimized media with amino acids, KSOMaa; Zenith Biotech). Embryos were cultured in KSOMaa in a $5 \% \mathrm{O}_{2}, 5 \% \mathrm{CO}_{2}, 90 \%$ $\mathrm{N}, 37^{\circ} \mathrm{C}$ humidified incubator.

Zygotes underwent a recovery period for a minimum of $1 \mathrm{~h}$ after collection before microinjection. Postinjection, mouse embryos were cultured for up to $120 \mathrm{~h}$ post siRNA injection in $\mathrm{KSOMaa}$ in a $5 \% \mathrm{O}_{2}, 5 \% \mathrm{CO}_{2}, 90 \% \mathrm{~N}, 37^{\circ} \mathrm{C}$ humidified incubator. For MEK inhibitor experiments, embryos were incubated in KSOMaa containing $1 \mu \mathrm{M}$ PD0325901 (Selleck Chemicals) or DMSO (vehicle control; Sigma-Aldrich) for $24 \mathrm{~h}$. For the in vitro blastocyst outgrowth assay, the zona pellucida was removed by briefly incubating blastocysts in Acidic Tyrode's Solution (Sigma-Aldrich). One to three blastocysts were transferred to one well of an eight-well chamber slide (Ibidi) on a feeder layer of irradiated mouse embryonic fibroblasts (ATCC, Canada). Embryos were cultured for 4 days in KO DMEM/F12 containing 20\% KO Serum Replacement (Life Technologies, Canada), 1\% GlutaMAX (Life Technologies), nonessential amino acids, $55 \mu \mathrm{M} \beta$-mercaptoethanol, and $1,000 \mathrm{U} / \mathrm{mL}$ mouse LIF (Sigma-Aldrich).

\section{Cytoplasmic microinjection of siRNA}

Short interfering RNA (siRNA) oligonucleotide sequences targeting the mouse p66Shc transcript and scrambled control targets were designed by us (see Table 1 for sequences), then synthesized by Thermo Fisher Scientific (Silencer Select siRNA, Canada). SiRNA were stored at $-20^{\circ} \mathrm{C}$ as a $100 \mu \mathrm{M}$ stock and diluted in nuclease-free water. Diluted siRNA solutions were back filled into Femtotips (outside diameter $1 \mu \mathrm{M}$; Eppendorf, Germany). Mouse zygotes were held in M2 medium overlaid with embryo culture grade oil (Zenith Biotech) during injection periods. The cytoplasm of mouse zygotes was injected with $10 \mathrm{pl}$ of $50 \mu \mathrm{M}$ of either p66Shcspecific or scrambled sequence siRNA using a FemtoJet Microinjector (Eppendorf) at the following settings: injection pressure $75 \mathrm{hPa}$, injection time $0.1 \mathrm{~s}$, and constant pressure $15 \mathrm{hPa}$ [15]. After injection, mouse zygotes were placed into KSOMaa and any embryos that had lysed were immediately removed from culture. Twenty zygotes were injected at a time, then moved to a $5 \% \mathrm{CO}_{2}, 37^{\circ} \mathrm{C}$ humidified incubator, until all injections were completed.

\section{Mouse embryonic stem cell transfection}

R1 mouse embryonic stem cells (Sick Kids, Toronto, Canada) were cultured without mouse embryonic fibroblast

TABle 1. SiRNA Sequences

\begin{tabular}{cl}
\hline p66Shc & Sense: UGAGUCUCUGUCAUCGCUGtt \\
siRNA & Antisense: CAGCGAUGACAGAGACUCAtt \\
No. 1 & \\
Scrambled & Sense: AUGCGUCGCGAUUAUAUCUtt \\
siRNA & Antisense: AGAUAUAAUCGCGACGCAUgc \\
No. 1 & \\
p66Shc & Sense: ACAACCCACUUCGGAAUGAtt \\
siRNA & Antisense: UCAUUCCGAAGUGGGUUGUac \\
No. 2 & \\
Scrambled & Sense: AGCCGUCAAUCACUACAGUtt \\
siRNA & Antisense: ACUGUAGUGAUUGACGGCUUc \\
No. 2 &
\end{tabular}


feeders on $0.1 \%$ gelatin in KO DMEM/F12/Neurobasal media (Life Technologies) supplemented with $1 \%$ N2, $2 \%$ B27 (Life Technologies), 1,000 U/mL mouse LIF (Esgro; Millipore), $3 \mu \mathrm{M}$ CHIR99021 (Selleck Chemicals), and $1 \mu \mathrm{M}$ PD0325901 (Selleck Chemicals). Cells were transfected with 60 pmol of siRNA with Lipofectamine 3000 (Thermo Fisher Scientific) according to manufacturer's protocols. Twentyfour hours post siRNA transfection, cells were lysed in radioimmunoprecipitation assay (RIPA, $150 \mathrm{mM} \mathrm{NaCl}, 1 \%$ Triton X-100, 0.5\% sodium deoxycholate, $0.1 \%$ SDS, $50 \mathrm{mM}$ Tris) buffer for immunoblotting.

\section{Quantitative real time reverse transcription-PCR}

RNA was extracted from pools of 20 cleavage stage embryos or 10 blastocysts using the PicoPure RNA Isolation Kit (Thermo Fisher Scientific) according to manufacturer's instructions. During extraction, RNA was incubated with RNaseFree DNase I (Qiagen, Canada) to digest genomic DNA from the samples. RNA was reverse transcribed to cDNA using SuperScript III (Thermo Fisher Scientific) according to the manufacturer's instructions with the following modifications: priming was done with random hexamers (Thermo Fisher Scientific) and a gene-specific reverse primer [14]. Quantitative real-time PCR was performed on a CFX384 thermal cycler (Bio-Rad, Canada) using SensiFAST SYBR (FroggaBio, Canada) and a final concentration of $400 \mathrm{nM}$ primers with the following cycling conditions: $95^{\circ} \mathrm{C}$ for $2 \mathrm{~min}, 39$ cycles of $95^{\circ}$ for $5 \mathrm{~s}, 60^{\circ} \mathrm{C}$ for $10 \mathrm{~s}$, and $72^{\circ} \mathrm{C}$ for $20 \mathrm{~s}$. Ct values were obtained from Bio-Rad CFX Manager 3.1 (Bio-Rad). Relative transcript abundance was determined by using the delta-delta $\mathrm{Ct}$ method using the geometric mean of $\mathrm{Ct}$ values of Ppia and Hprt for normalization. Primer sequences are found in Table 2.

\section{Immunoblotting}

Cells and embryos were lysed in RIPA buffer containing protease and phosphatase inhibitors (Millipore) and stored at $-80^{\circ} \mathrm{C}$ until processing. Total protein lysates were resolved on a 4\%-12\% Bis-Tris polyacrylamide gel (Thermo Fisher Scientific) and transferred to a PVDF membrane (Millipore).
Membranes were blocked in either 5\% skim milk in TBST for $1 \mathrm{~h}$ at room temperature. Membranes were then incubated in primary antibody diluted in 5\% skim milk in TBST overnight at $4^{\circ} \mathrm{C}$ at the following dilutions: mouse anti-Shc (610879, 1:500; BD Biosciences), rabbit anti-CDX2 (ab76541, 1:500; Abcam), and rabbit anti-EOMES (ab23345, 1:1,000; Abcam). Membranes were then incubated in HRPconjugated secondary antibody (1:2,000; Jackson Laboratories) diluted in 5\% skim milk or BSA in TBST for $1 \mathrm{~h}$ at room temperature before ECL detection (Luminata Forte; Millipore). Western blot images were taken with a ChemiDoc Imaging System (Bio-Rad) and densitometry analysis of band intensity was performed in ImageLab 4.0 (Bio-Rad). Membranes were then stripped for 20 min using Antibody Stripping Buffer (FroggaBio), then blocked and probed with mouse anti- $\beta$-actin-HRP (A3854, 1:10,000; Sigma).

\section{Immunofluorescence and confocal microscopy}

Embryos were fixed in $2 \%$ paraformaldehyde in PBS for $30 \mathrm{~min}$ at room temperature. Embryos were then permeabilized in $0.25 \%$ Triton X-100 in PBS for 30 min, blocked in 5\% normal goat serum in PBS, and incubated overnight at $4{ }^{\circ} \mathrm{C}$ in the following primary antibodies: mouse anti-NT-Shc (AM00143PU-N, 1:100, validated in Edwards et al. [14]; Acris), mouse anti-OCT3/4 (C-10, sc-5279, 1:50, Nichols et al. [7]; Santa Cruz Biotech), goat anti-GATA4 (sc-1237, 1:200, Nichols et al. [7]; Santa Cruz Biotech), rabbit anticleaved caspase-3 (AF835, 1:50; R\&D Systems), rabbit antiCDX2 (1:100, [18]; Abcam), rabbit anti-NANOG (RCAB001P, 1:200; ReproCell), goat anti-SOX17 (AF1924, 1:100; R\&D Systems), and rabbit anti-DUSP4 (ab216576, 1:200; Abcam). Embryos were then incubated at room temperature for $1 \mathrm{~h}$ in either Alexa Fluor 488- or 594-conjugated secondary antibodies (Thermo Fisher Scientific), and mounted in VECTASHIELD Antifade solution (Vector Laboratories) on a glass microscopy slide. Confocal microscopy was performed with a Zeiss LSM510 laser scanning confocal microscope with $10 \mathrm{z}$-sections taken through each blastocyst (mean z-stack step size $=6.83 \mu \mathrm{M}, \max =9.324 \mu \mathrm{M}$,

Table 2. Primer Sequences

\begin{tabular}{|c|c|c|}
\hline Gene & Sequences & Expected product size, $b p$ \\
\hline p66Shc & $\begin{array}{l}\text { F: 5'-CCGACTACCCTGTGTTCCTTCTT-3' } \\
\text { R: 5'-CCCATCTTCAGCAGCCTTTCC-3' }\end{array}$ & 111 \\
\hline Cdknla & $\begin{array}{l}\text { F: 5'-TCCAGACATTCAGAGCCACAGG-3' } \\
\text { R: 5'-ACGGGACCGAAGAGACAACG-3' }\end{array}$ & 97 \\
\hline$R b 1$ & $\begin{array}{l}\text { F: 5'-CTTGCATGGCTTTCAGATTCACCT-3' } \\
\text { R: } 5^{\prime} \text {-ATGGTTACCCTGGAGAGGCAG-3' }\end{array}$ & 117 \\
\hline $\operatorname{Trp} 53$ & $\begin{array}{l}\text { F: 5'-GGACCATCCTGGCTGTAGGT-3' } \\
\text { R: 5'-GGCAGTCATCCAGTCTTCGG-3' }\end{array}$ & 113 \\
\hline Gadd45a & $\begin{array}{l}\text { F: 5'-TGCTGGTGACGAACCCACAT-3' } \\
\text { R: 5'-CATGTAGCGACTTTCCCGGC-3' }\end{array}$ & 86 \\
\hline Dusp4 & $\begin{array}{l}\text { F: 5'-AAACTGGGTGCCGTTCAGAT-3' } \\
\text { R: 5'-AACACCCAATGTATCCGCGA-3' }\end{array}$ & 116 \\
\hline Ppia & $\begin{array}{l}\text { F: 5'-GTCCTGGCATCTTGTCCATG-3' } \\
\text { R: 5'-TGCCTTCTTTCACCTTCCCA-3' }\end{array}$ & 126 \\
\hline Hprt & $\begin{array}{l}\text { F: 5'-GCTTACCTCACTGCTTTCCG-3' } \\
\text { R: 5'-ATCATCGCTAATCACGACGC-3' }\end{array}$ & 128 \\
\hline
\end{tabular}


$\min =4.957 \mu \mathrm{M})$. Laser settings were unchanged when detecting the same primary antibody between treatment groups. Nuclei with detectable antibody staining were manually counted in duplicate in ImageJ (National Institutes of Health). For fluorescence intensity quantification of p66Shc knockdown, 3D z-stack projections were created in ZEN Black (Zeiss, Germany) exported as TIFF files and opened in ImageJ. Images were converted to grayscale and LUT inverted. The mean gray value was measured, and the average mean gray value of three images of embryos with no primary antibody was subtracted to control for background fluorescence. To quantify OCT3/4 immunofluorescence intensity in blastocysts, individual z-stacks were analyzed by Modular Interactive Nuclear Segmentation (MINS, [19] and the fluorescence intensity of OCT3/4 of nuclei detected by the software was determined and averaged.

\section{BrdU incorporation assay}

Embryos were incubated in KSOMaa containing $10 \mu \mathrm{M}$ of bromodeoxyuridine (BrdU; Acros Organics) for $45 \mathrm{~min}$ before fixation with $2 \%$ paraformaldehyde. Embryos were then processed for immunofluorescence as described above with the following additions: after permeabilization, embryos were incubated in $300 \mu \mathrm{g} / \mathrm{mL}$ DNase for $30 \mathrm{~min}$ at $37^{\circ} \mathrm{C}$ [20]. Embryos were incubated overnight at $4^{\circ} \mathrm{C}$ in mouse anti-BrdU antibody (G3G4, 1:100; Developmental Studies Hybridoma Bank deposited by Kaufman, S.J.), then incubated at room temperature for $1 \mathrm{~h}$ in goat anti-mouse Alexa Fluor 488 (1:400). Confocal microscopy was performed as described above.

\section{Statistical analyses}

Statistical tests were performed using Prism 6 (GraphPad Inc.) for Students' $t$-test (unpaired, two-tailed with equal variance) or Fisher's exact test (two-tailed). At least three independent replicates were performed for each experiment.

\section{Results}

\section{Specific knockdown of p66Shc in mouse preimplantation embryos}

To target p66Shc for knockdown by RNAi, two different siRNA sequences were designed to recognize the unique $\mathrm{N}$ terminal exon of the p66Shc transcript variant of mouse Shc1 (sequences in Table 1). Fifty micrometer of each sequence, designated Nos. 1 and 2, and its associated control (scrambled nucleotide) sequence was microinjected into the cytoplasm of zygotes. Pools of embryos were then collected at different time points post siRNA injection to assess knockdown efficiency by quantitative real-time reverse transcription-PCR. We were unable to detect a statistically significant decrease in p66Shc transcript abundance $24 \mathrm{~h}$ post siRNA No. 1 injection due to the high variability of p66Shc transcript levels in control 2-cell embryos [14] (Fig. 1A). However, p66Shc transcript abundance significantly decreased to $33 \%$ of control levels in embryos injected with p66Shc siRNA No. $148 \mathrm{~h}$ postinjection $(P<0.05$, Fig. 1A). By 72 and $96 \mathrm{~h}$ postinjection, p66Shc transcript abundance levels in embryos injected with p66Shc siRNA became comparable to controls (Fig. 1A). P66Shc siRNA sequence No. 2 also significantly knocked down p66Shc transcript abundance $48 \mathrm{~h}$ post siRNA injection (Supplementary
Fig. S1A; Supplementary Data are available online at www.liebertpub.com/scd). Due to the higher knockdown efficiency at $48 \mathrm{~h}$ postinjection, the remaining experiments were performed with sequence No. 1. Some experiments were repeated using siRNA sequence No. 2 to confirm gene-specific phenotypes (eg, Supplementary Fig. S3).

To confirm knockdown at the protein level, zygotes were injected with siRNA No. 1 sequences, fixed at 24, 48, and $72 \mathrm{~h}$ postinjection, and stained for p66Shc immunofluorescence. Significant decreases in p66Shc fluorescence intensity were detected at all three time points post siRNA injection, with the greatest reduction in fluorescence intensity observed at $48 \mathrm{~h}$ post siRNA injection (29\% of control levels, $P<0.0001$, Fig. 1B, additional microscopy pictures in Supplementary Fig. S2). Thus, our results demonstrate that $50 \mu \mathrm{M}$ of p66Shcspecific siRNA significantly reduces p66Shc transcript and protein levels during mouse preimplantation development. Knockdown specificity and efficiency was confirmed in R1 mouse embryonic stem cells (Supplementary Fig. S1B).

\section{P66Shc knockdown embryos form blastocysts containing more TE cells}

We next determined if embryos receiving p66Shc siRNA could form blastocysts. Blastocyst formation did not significantly change in embryos receiving p66Shc siRNA compared with controls with frequencies of blastocyst development observed between $60 \%$ and $70 \%$ of all cultured 2-cell embryos (Fig. 2A) and no significant changes in cavitation (Fig. 2B). Blastocysts formed by embryos that received p66Shc siRNA were morphologically similar to scrambled siRNA-injected controls (Fig. 2C). However, p66Shc knockdown blastocysts contained, on average, 12 more cells than scrambled controls at $96 \mathrm{~h}$ postinjection $(\sim 70$ cells in p66Shc knockdown embryos compared with 58 in scrambled controls, Fig. 2D, middle panel). Embryos not injected with siRNA formed blastocysts with an average of 64 cells after $96 \mathrm{~h}$ of culture, suggesting that introduction of siRNA oligonucleotides does not significantly affect cell viability in the embryo (Supplementary Fig. S3A). Interestingly, p66Shc knockdown embryos at $72 \mathrm{~h}$ postinjection contained significantly fewer cells than scrambled controls, whereas p66Shc knockdown embryos at $120 \mathrm{~h}$ postinjection contained cell numbers comparable to controls (Fig. 2D). Our results thus demonstrate that although embryos with p66Shc knockdown can form blastocysts, they produce blastocysts with more total cells than controls 4 days after p66Shc siRNA introduction.

Due to p66Shc knockdown affecting blastocyst total cell number, we next investigated its effects on cell apoptosis and proliferation at $96 \mathrm{~h}$ post siRNA injection. There was no significant difference in the percentage of cleaved caspase-3positive cells in p66Shc knockdown blastocysts compared with controls, suggesting that apoptosis is not contributing to the change in total cell number (Fig. 3A). However, p66Shc knockdown blastocysts at $96 \mathrm{~h}$ displayed significantly increased BrdU incorporation, suggesting that altered proliferation may lead to the increased total cell number observed (Fig. 3A). When stained for markers of the TE (CDX2) and ICM (OCT3/4, SOX2), we observed that p66Shc knockdown blastocysts had on average more CDX2-positive cells compared with scrambled controls, whereas the average number of OCT3/4-positive cells was comparable between groups 
A

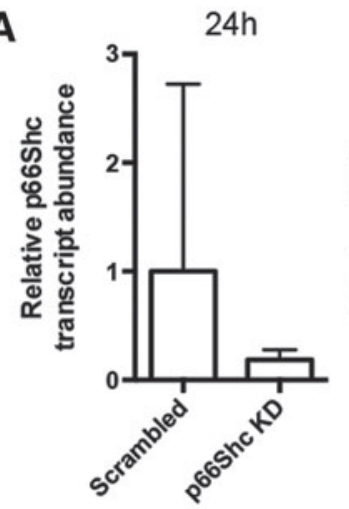

B

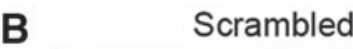

$48 \mathrm{~h}$

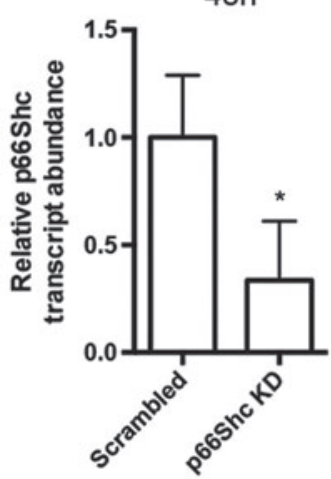

$72 \mathrm{~h}$

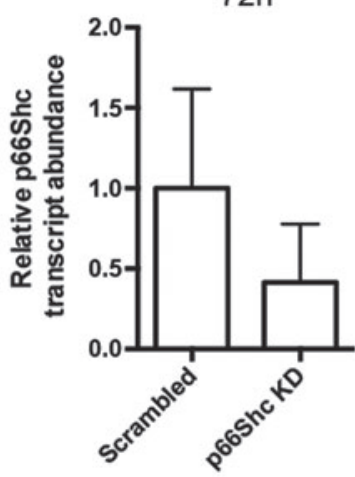

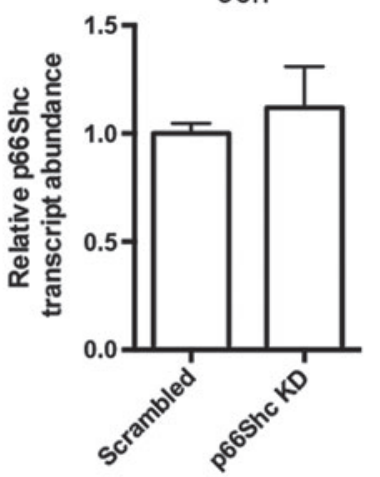

p66Shc KD
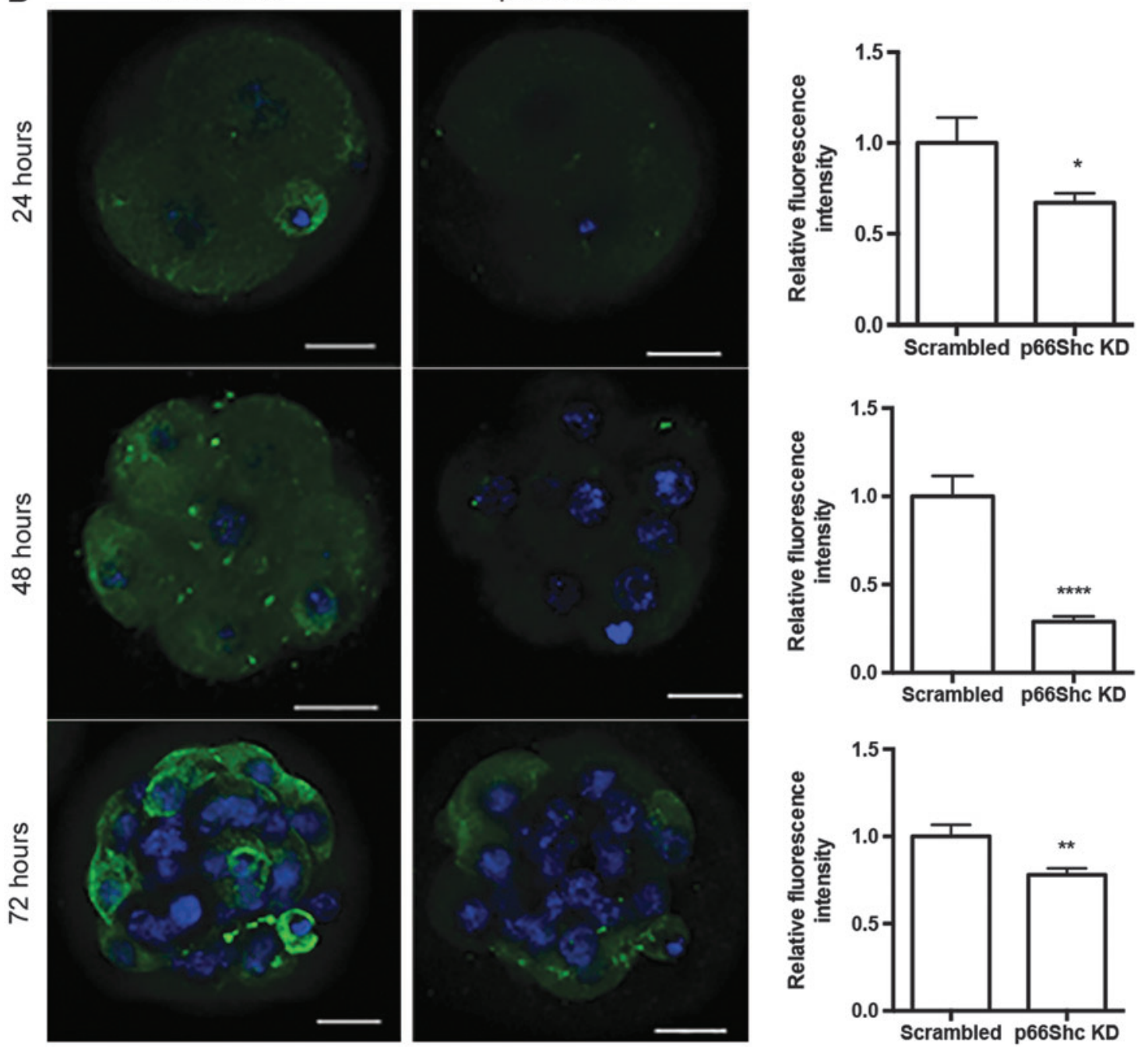

FIG. 1. p66Shc siRNA reduces p66Shc transcript and protein abundance in mouse preimplantation embryos. (A) RTqPCR was performed on pools of 20 embryos or 10 blastocysts at four time points postinjection of $50 \mu \mathrm{M}$ siRNA $(24 \mathrm{~h} N=5$ pools of embryos, $48 \mathrm{~h} N=4,72 \mathrm{~h} N=4,96 \mathrm{~h} N=3$ ). P66Shc transcript abundance is significantly reduced at $48 \mathrm{~h}$ post siRNA injection (mean $\pm \mathrm{SD}, * P<0.05$, Student's $t$-test). (B) Immunofluorescence and confocal microscopy for p66Shc protein was performed on embryos taken at three time points post siRNA injection. The relative fluorescence intensities of Z-stack projections were quantified. P66Shc fluorescence intensity was significantly decreased at $24(n=15$ embryos), 48 $(n=17)$, and $72 \mathrm{~h}(n=14)$ post p66Shc-siRNA injection compared with controls (mean $\pm \mathrm{SEM}, * P<0.05, * * P<0.01$, $* * * * P<0.00001$, Student's $t$-test). Scale bars are $20 \mu \mathrm{m}$. RT-qPCR, reverse transcription-quantitative polymerase chain reaction; siRNA, short interfering RNA. 
A

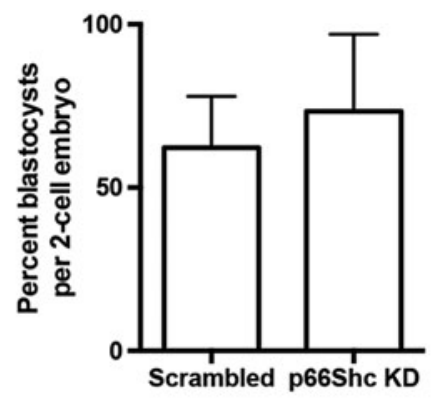

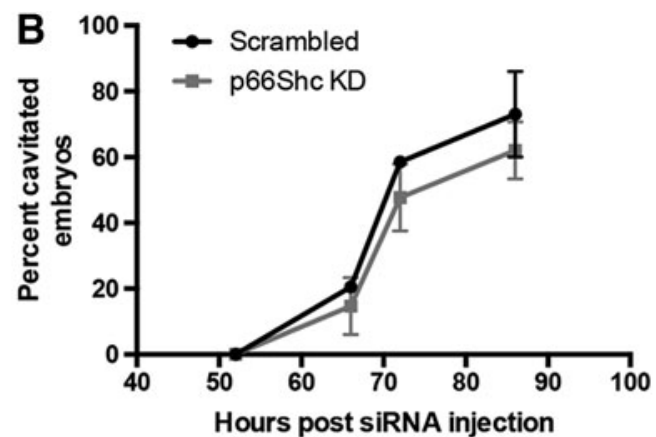

Hours post siRNA injection

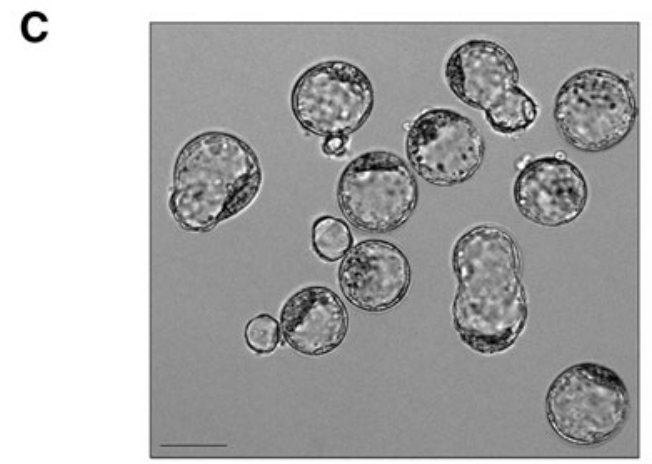

Scrambled

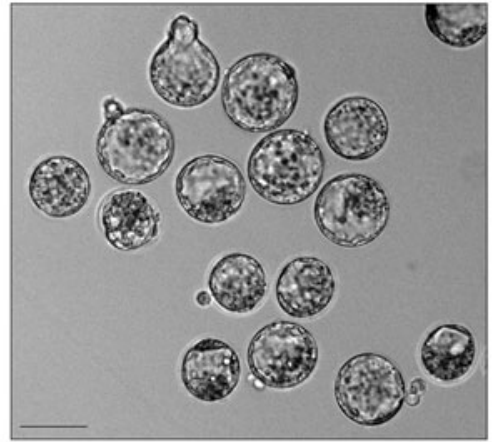

p66Shc KD
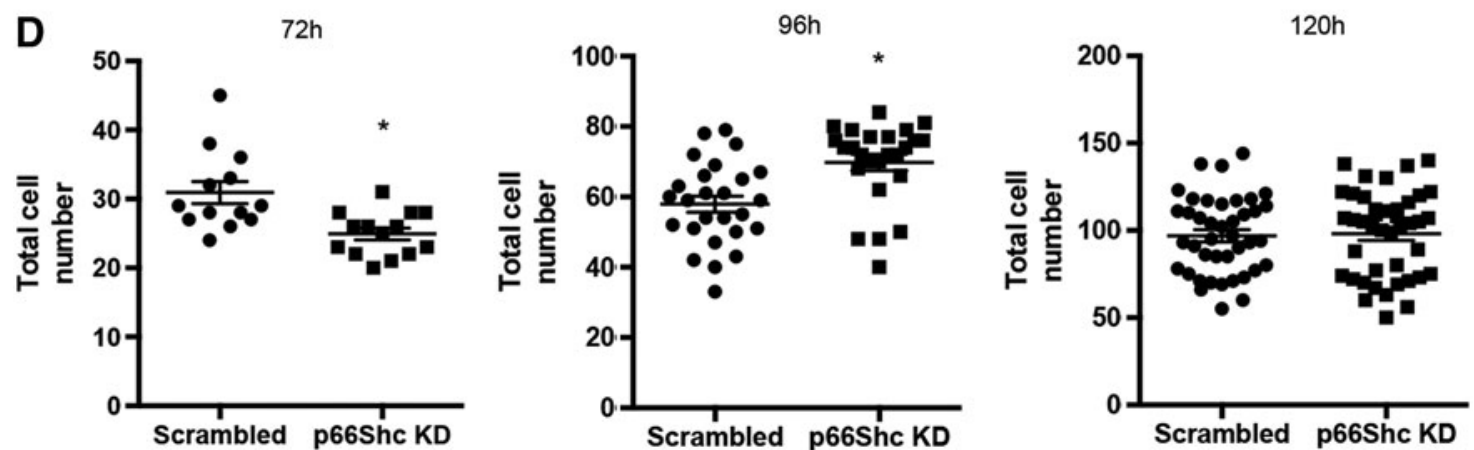

FIG. 2. p66Shc knockdown embryos form blastocysts containing more cells than controls. (A) Embryos receiving p66Shc siRNA form blastocysts at equivalent rates to scrambled controls $(N=3$ experimental replicates, mean \pm SD). (B) p66Shc knockdown embryos form blastocyst cavities at similar rates to scrambled controls $(N=3$ pools of 14 embryos, mean \pm SEM). (C) p66Shc knockdown embryos form morphologically similar blastocysts compared with scrambled controls $(\mathrm{scale} b a r s=100 \mu \mathrm{m})$. (D) Total cell number in p66Shc knockdown embryos is significantly lower than controls at $72 \mathrm{~h}$ post siRNA injection $(n=15$ per group, $* P<0.05$, Student's $t$-test), significantly higher than controls at $96 \mathrm{~h}$ post siRNA injection $(n=25$ per group, $* P<0.05$, Student's $t$-test) and not significantly different at $120 \mathrm{~h}$ post siRNA injection ( $n=41$ per group). Graphs are the mean \pm SEM.

(Fig. 3B). We confirmed in an independent experiment that the number of inner cells is unchanged in p66Shc knockdown blastocysts compared with scrambled controls by SOX2 immunostaining (Fig. 3C). Therefore, the increase in total cell number of p66Shc knockdown blastocysts is likely due to altered proliferation of the TE and not the ICM. Interestingly, p66Shc knockdown blastocysts had significantly reduced $R b 1$ transcript abundance compared with controls, suggesting that the cell cycle may be altered in these embryos (Fig. 3D).

\section{P66Shc knockdown accelerates the onset of PE marker expression in mouse blastocysts}

Although p66Shc knockdown embryos formed blastocysts at the same frequency and appeared morphologically similar to controls, we next investigated the effects of p66Shc knockdown on cell differentiation to the TE and ICM lineages. To investigate this directly, we processed blastocysts $96 \mathrm{~h}$ post siRNA injection for immunofluorescence and confocal microscopy for CDX2 and OCT3/4, definitive markers of the TE and ICM, respectively. Some blastocysts contained cells positive for both CDX2 and OCT3/4, which is consistent with the finding that all cells of the early blastocyst initially express OCT3/4 before its expression is restricted to the inner cells [21]. Notably, 60\% of p66Shc knockdown blastocysts showed OCT3/4 restriction to the inner cells compared with $25 \%$ of scrambled control blastocysts when fixed at $96 \mathrm{~h}$ post siRNA injection (Fig. 4A, B). To quantify this difference in staining pattern, we determined the total cell number and categorized embryos from both groups into blastocysts containing 32-64 cells or blastocysts 

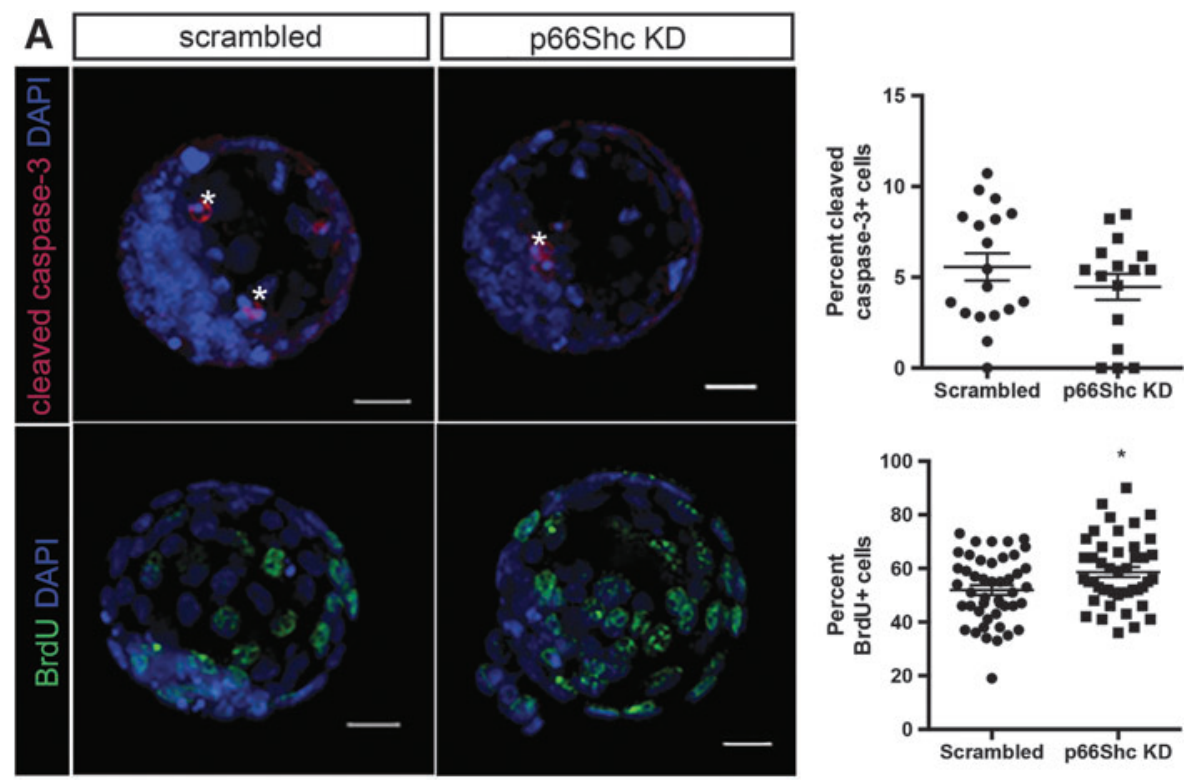

B
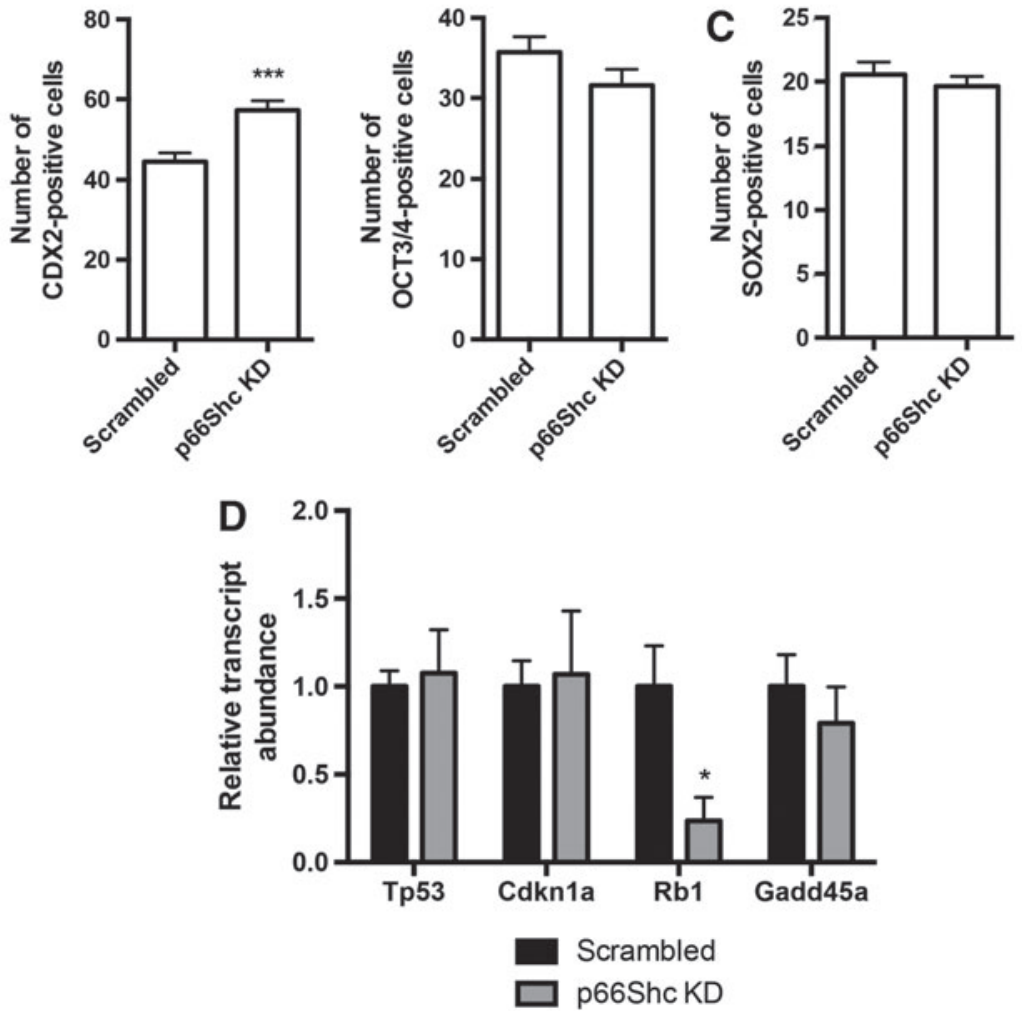

FIG. 3. Altered cell proliferation in p66Shc knockdown blastocysts. (A) p66Shc knockdown blastocysts have similar levels of cleaved caspase-3 cells compared with controls $(n=16$, mean \pm SEM, white asterisks indicate cells positive for cleaved caspase-3). However, p66Shc knockdown blastocysts have a significantly increased percentage of cells positive for BrdU incorporation after $45 \mathrm{~min}$ of incubation compared with controls $(n=46$, $* P<0.05$, mean \pm SEM, Student's $t$-test). (B) p66Shc knockdown blastocysts have a significant increase in the number of CDX2-positive cells compared with scrambled controls, whereas the number of OCT3/4positive cells is unchanged between groups $(n=26$, mean \pm SEM, $* * * P<0.001$, Students' $t$-test). (C) p66Shc knockdown blastocysts have similar numbers of SOX2-positive cells compared with scrambled controls $(n=33$, mean \pm SEM, Students' $t$-test). (D) RT-qPCR was performed on pools of 10 blastocysts for transcript abundance of cell cycle markers. P66Shc knockdown blastocysts have significantly lower $R b 1$ transcript abundance compared with controls $(N=3$ pools of 10 embryos, $* P<0.05$, mean $\pm \mathrm{SD}$, Student's $t$ test). containing 64-128 cells, then counted the number of cells positive for both CDX2 and OCT3/4 (double positive). In 32-64-cell blastocysts, p66Shc knockdown blastocysts contained approximately the same percentage of double-positive nuclei. However, at the 64-128-cell stage, p66Shc knockdown embryos contained a significantly lower percentage of double-positive nuclei, supporting the observation that OCT3/4 expression is restricted earlier in p66Shc knockdown blastocysts compared with controls (Fig. 4C, D).

We observed that OCT3/4 fluorescence intensity appeared to be elevated in the inner cells of 32-64-cell p66Shc knockdown blastocysts compared with scrambled controls. Quantification of OCT3/4 fluorescence intensity revealed that on average, nuclei of p66Shc knockdown blastocysts had significantly increased OCT3/4 immunofluorescence intensity than scrambled controls (Fig. 4E). This difference is lost in 64-128-cell blastocysts, suggesting that there is a transient window in which OCT3/4 expression is elevated in the inner cells of p66Shc knockdown blastocysts (Fig. 4F). In contrast, protein abundance of CDX2 and EOMES determined by immunoblotting were unaffected by p66Shc knockdown, suggesting that p66Shc knockdown does not 

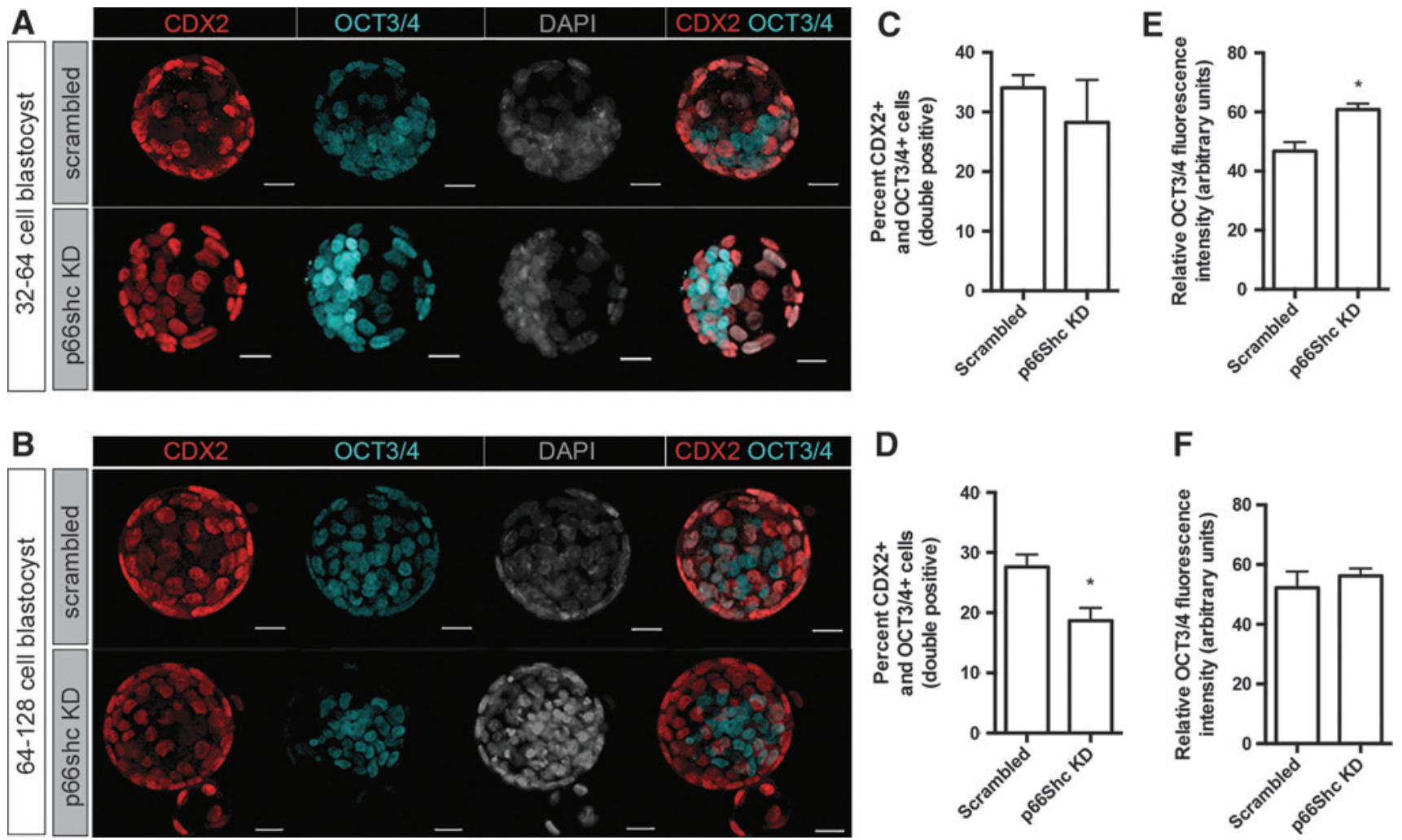

FIG. 4. Elevated OCT3/4 levels and earlier restriction of OCT3/4 to the inner cells in p66Shc knockdown blastocysts. Embryos were categorized according to cell number before analysis: $32-64$ cells (scrambled mean $\pm \mathrm{SD}=51.94 \pm 8.33$, p66Shc KD mean $=49.60 \pm 7.93$ ) or 64-128 cells $($ scrambled mean $=71.38 \pm 5.48$, p66Shc KD mean $=74.76 \pm 4.46)$. $(\mathbf{A})$ Representative confocal microscopy images of scrambled and p66Shc knockdown 32-64-cell blastocysts immunostained for CDX2 and OCT3/4. Scale bars are $20 \mu \mathrm{m}$. (B) Representative confocal microscopy images of scrambled and p66Shc knockdown 64-128-cell blastocysts. Scale bars are $20 \mu \mathrm{m}$. (C) p66Shc knockdown blastocysts have similar CDX2-positive/ OCT3/4-positive nuclei compared with scrambled controls in 32-64-cell blastocysts ( $n=19$, mean \pm SEM, Students' $t$-test). (D) p66Shc knockdown blastocysts have significantly fewer CDX2-positive/OCT3/4-positive nuclei in 64-128-cell blastocyst $(n=23, * P<0.05$, mean \pm SEM, Student's $t$-test). (E) p66Shc knockdown blastocysts have significantly higher nuclear OCT3/4 fluorescence intensity compared with scrambled controls in 32-64-cell blastocysts $(n=19$, *P<0.05, mean \pm SEM, Student's $t$-test). (F) p66Shc knockdown blastocysts have no significant changes in nuclear OCT3/4 fluorescence intensity compared with scrambled controls $(n=23$, mean \pm SEM). KD, knockdown.

affect the relative abundance of TE markers in the blastocyst (Supplementary Fig. S3B). Our results thus demonstrate that p66Shc knockdown causes altered proliferation in the TE, increased OCT3/4 expression in the ICM of early blastocysts, and faster restriction of OCT3/4 to the inner cells of the blastocyst.

Since OCT3/4 is restricted earlier to the inner cells in p66Shc knockdown blastocysts, we hypothesized that the ICMs in these embryos might be more developmentally advanced with regard to their differentiation program and may be specifying EPI versus PE fate earlier than controls. Furthermore, increased OCT3/4 expression is associated with endoderm differentiation in blastocysts and embryonic stem cells [22,23]. To investigate this, blastocysts were fixed and processed for immunofluorescence for GATA4 and NANOG, markers of the PE and EPI fates, respectively (Fig. 5A). In 32-64-cell p66Shc knockdown blastocysts, we observed that the percentage of total cells and inner cells expressing GATA4 was significantly higher than that for scrambled controls (Fig. 5B and Supplementary Fig. S3C). This increase in the GATA4-positive cell proportion of in- ner cells was at the expense of NANOG-positive cells, and not due to selective proliferation of GATA-positive cells, as the total number of inner cells was unchanged between knockdown and control blastocysts (Fig. 5C, D). The percentage of GATA4-positive cells of total cells was also significantly increased in embryos receiving p66Shc siRNA sequence No. 2, verifying a gene-specific phenotype (Supplementary Fig. S3C). To further confirm our findings, we immunostained blastocysts for SOX17, an earlier marker of PE specification (Fig. 5E). We observed that like GATA4, the percentage of inner cells expressing SOX17 was significantly increased in p66Shc knockdown blastocysts compared with scrambled controls (Fig. 5F).

As ICM cells are not committed until the late blastocyst stage [5], we investigated whether the increase in cells adopting a PE fate in p66Shc knockdown blastocysts persisted in late blastocysts. Blastocysts were cultured up to $120 \mathrm{~h}$ post siRNA injection, then fixed and processed for GATA4, SOX17, and NANOG immunofluorescence. We observed a significant increase in the percentage of GATA4positive cells of total inner cells and a decreased percentage 

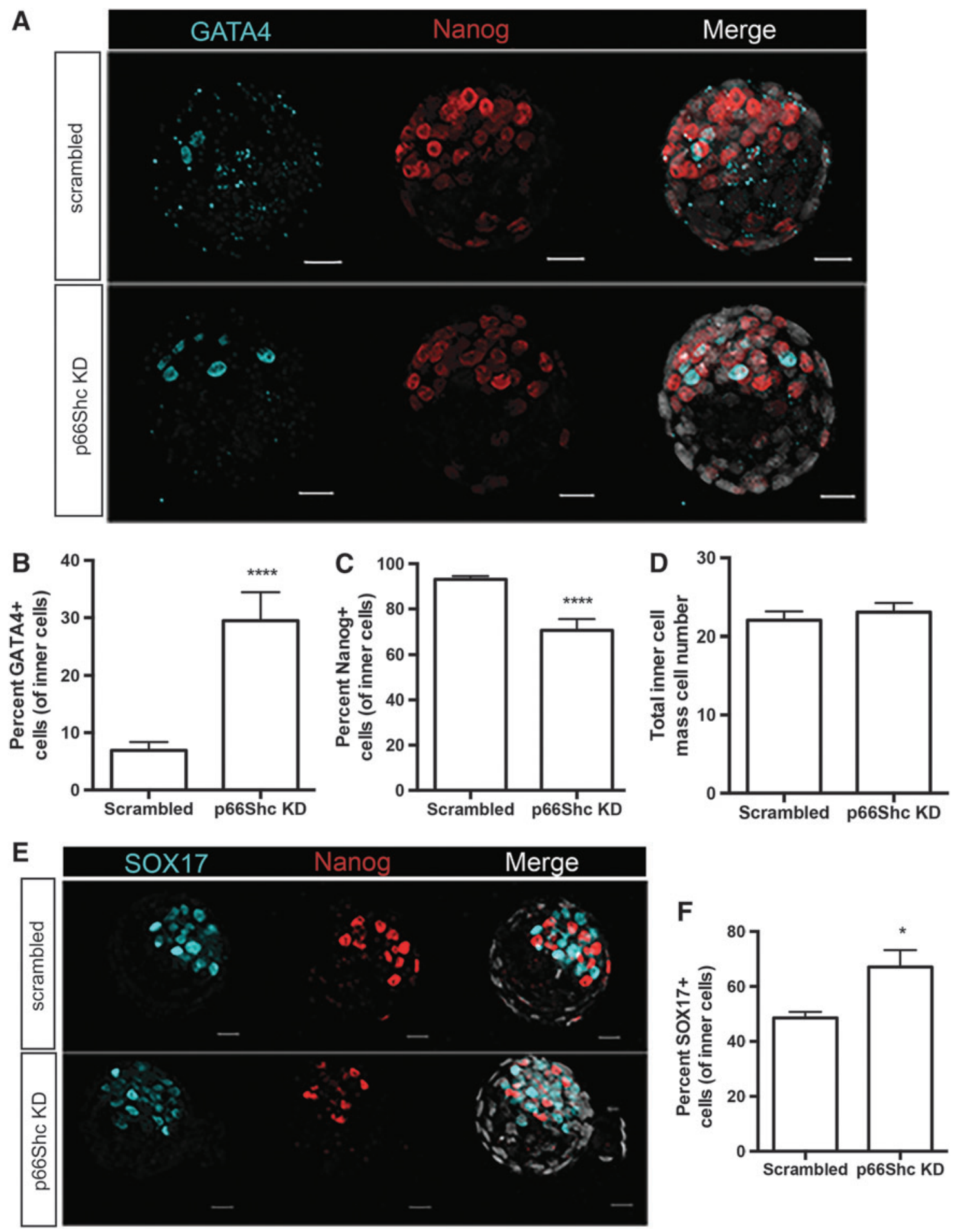

FIG. 5. Knockdown of p66Shc increases the number of cells expressing PE markers in the inner cell mass of 32-64-cell blastocysts (A) Blastocysts were fixed and processed for immunofluorescence and confocal microscopy 96h post siRNA injection. Panels display representative confocal microscopy images. Scale bars are $20 \mu \mathrm{m}$. P66Shc knockdown blastocysts have significantly increased percentage of inner cells positive for GATA4 (B) and significantly decreased percentage positive for NANOG (C) $(n=19$ per group, mean \pm SEM, $* * * * P<0.0001$, Student's $t$-test $)$. (D) The total cell number of the inner cell mass was not significantly different between p66Shc knockdown embryos and controls $(n=19$ per group, mean \pm SEM, Student's $t$-test). (E) Blastocysts were processed for immunofluorescence for SOX17 and NANOG. Panels display representative confocal microscopy images. Scale bars are $20 \mu \mathrm{m}$. (F) The percentage of SOX17 cells of total inner cells is significantly increased in p66Shc knockdown blastocysts compared with scrambled controls $(n=22$, mean \pm SEM, $* P<0.05$, Student's $t$-test). PE, primitive endoderm. 
of NANOG-positive cells in p66Shc knockdown blastocysts (Fig. 6A, B). Additionally, the percentage of SOX17-positive cells of inner cells in late p66Shc knockdown blastocysts was significantly increased compared with scrambled controls (Fig. 6C). The total number of inner cells did not signifi- cantly change between groups, nor did it significantly increase from 32- to 64-cell blastocysts, suggesting that some GATA4-positive cells may have altered their fate to become NANOG-positive (Fig. 6D). Additionally, when blastocysts were categorized based on their GATA4 and NANOG
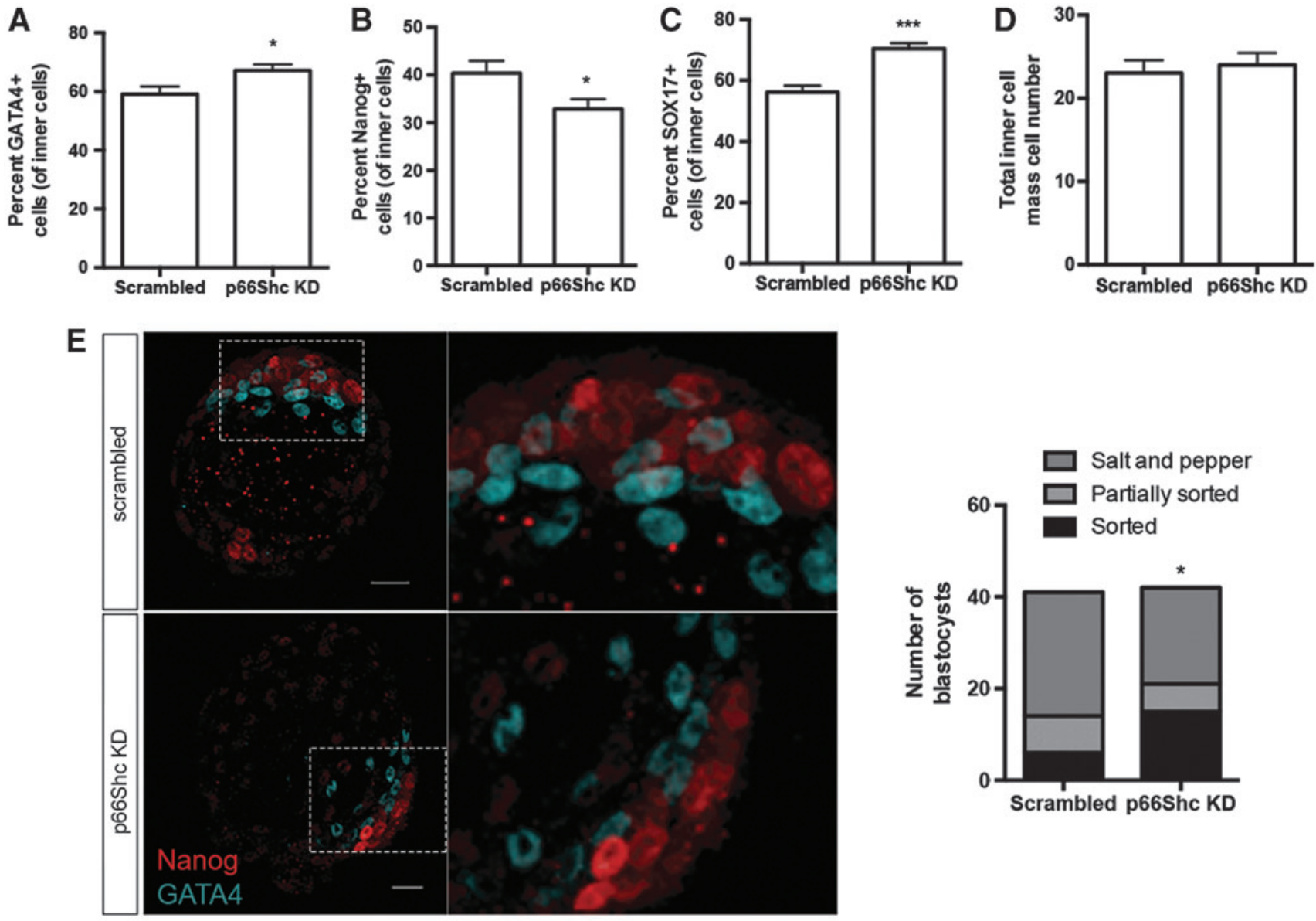

$\mathbf{F}$
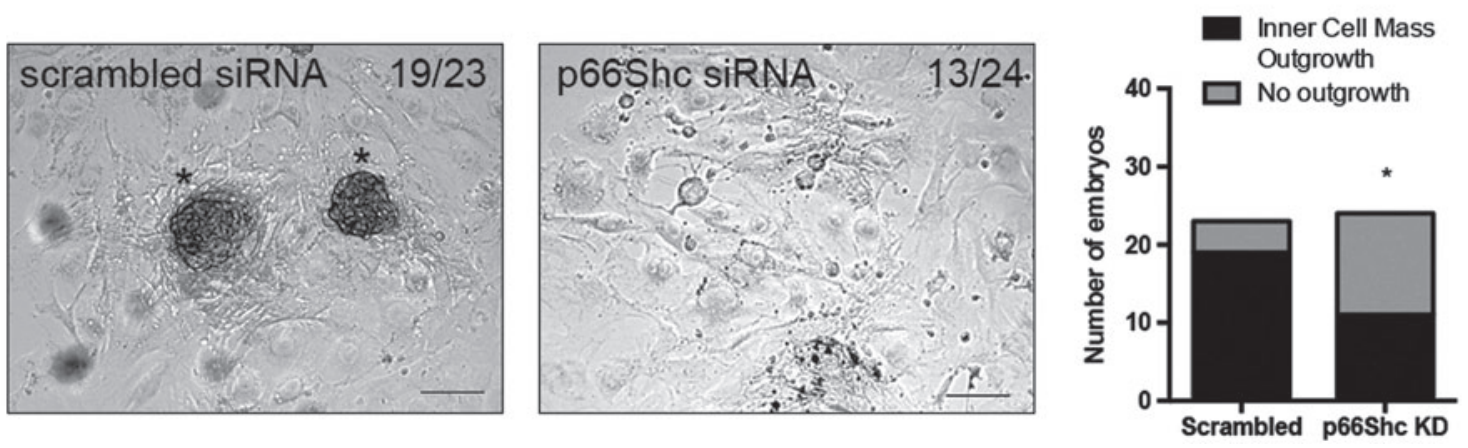

FIG. 6. Knockdown of p66Shc accelerates sorting of the PE in 64-128-cell blastocysts. (A) Blastocysts were processed for immunofluorescence and confocal microscopy $120 \mathrm{~h}$ post siRNA injection. p66Shc knockdown late blastocysts had a significant increase in the percentage of inner cells positive for GATA4 (B), a significant decrease in cells positive for NANOG (C) $(n=41$ blastocysts, mean \pm SEM, $* P<0.05$, Student's $t$-test), and a significant increase in the percentage of inner cells positive for SOX17 (C) $(n=22$ blastocysts, mean \pm SEM, $* * * P<0.001$, Student's $t$-test). (D) Total number of cells in the inner cell mass is not significantly different in p66Shc knockdown embryos compared to controls ( $n=41$ blastocysts). (E) In 64-128-cell embryos, p66Shc knockdown blastocysts also had a significantly higher proportion of embryos displaying sorted PE staining compared with scrambled controls ( $n=41$ blastocysts per group, ${ }^{*} P<0.05$, Fisher's exact test). Scale bars are $20 \mu \mathrm{m}$. (F) Bright field microscopy of in vitro blastocyst outgrowths. A significantly lower proportion of p66Shc knockdown blastocysts formed inner cell mass-derived outgrowths (asterisks) compared with scrambled controls ( $n=23$ and 24 outgrowths per group, $* P<0.05$, Fisher's exact test). Numbers in the image panels indicate the number of outgrowths resembling the displayed morphology. Scale bars are $100 \mu \mathrm{m}$. 
staining pattern to determine the extent of PE sorting [24], $36 \%$ of p66Shc knockdown blastocysts contained GATA4positive cells categorized as sorted, versus only $14 \%$ of the control blastocyst population containing sorted GATA4positive cells (Fig. 6E). Altogether, our results suggest that not only does the differences in EPI and PE cell proportions in p66Shc knockdown blastocysts persist until $120 \mathrm{~h}$ post siRNA injection, but these blastocysts also show much earlier sorting of PE cells to the PE layer.

We next determined if the reduction of NANOG-expressing cells in the ICMs of p66Shc knockdown blastocysts would negatively affect EPI survival in vitro. Blastocysts were plated in conditions supporting embryonic stem cell derivation (eg, EPI survival) for 5 days and then assessed for the presence of an ICM-derived outgrowth or cell mass (Fig. 6F). We observed that only $46 \%$ of p66Shc siRNA-injected blastocysts formed detectable ICM cell masses ( $n=11$ of 24$)$. Fifty-four percent of p66Shc knockdown blastocysts did not form an ICM-derived outgrowth from $72 \mathrm{~h}$ of culture onward, suggesting that the EPI population from these blastocysts did not survive ( $n=13$ of 24 , Supplementary Fig. S4A). ICM-derived outgrowths formed from scrambled blastocysts stained positive for OCT3/4 (EPI) and GATA4 (endoderm) ( $n=8$ of 8 ), whereas outgrowths from $\mathrm{p} 66 \mathrm{Shc}$ knockdown blastocysts with no ICM cell mass had disorganized and diffuse OCT3/4 and GATA4 immunostaining, suggesting that neither the EPI nor the PE survived during outgrowth formation ( $n=4$ of 7 , Supplementary Fig. S4B). The remaining three p66Shc knockdown outgrowths that formed an ICM clump stained positive for OCT3/4 and GATA4 staining, suggesting that some p66Shc knockdown blastocysts may have a critical number of NANOG-positive cells required for survival of the ICM in vitro, whereas the majority do not. Our results suggest that p66Shc knockdown in preimplantation embryos may affect the survival of the ICM in vitro.

\section{p66Shc knockdown increases the number of DUSP4-positive cells in the blastocyst}

As the adoption of PE fate in the ICM is dependent on FGF4/MAPK signaling [5,7], we next determined if decreased p66Shc levels resulted in a greater number of cells expressing PE markers to emerge due to increased or inappropriate MAPK signaling. Like previous studies [6], we were unable to detect phosphorylated-ERK1/2 in ICM cells by immunofluorescence. Although we attempted localization of total-ERK1/2 to detect nuclear translocation of ERK1/2, we encountered high background staining by
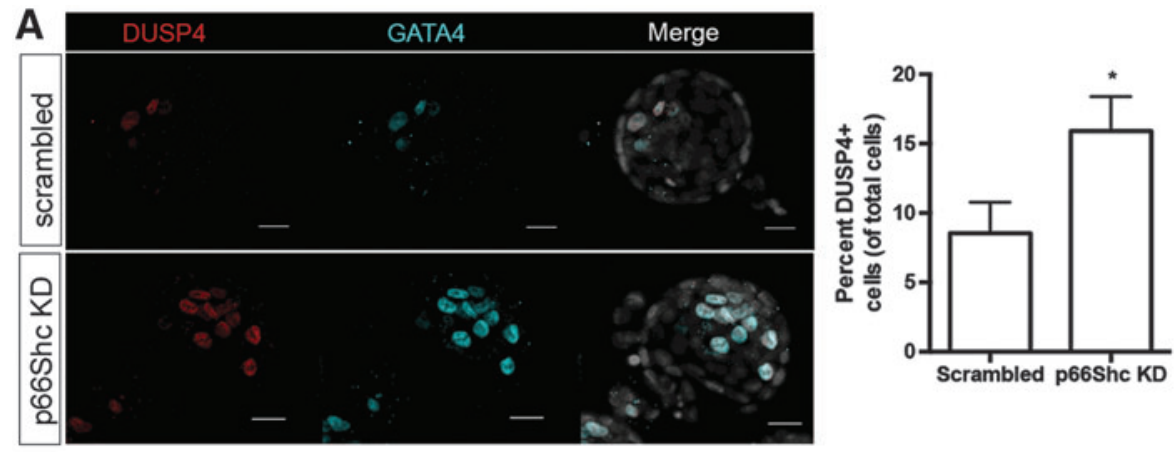

B

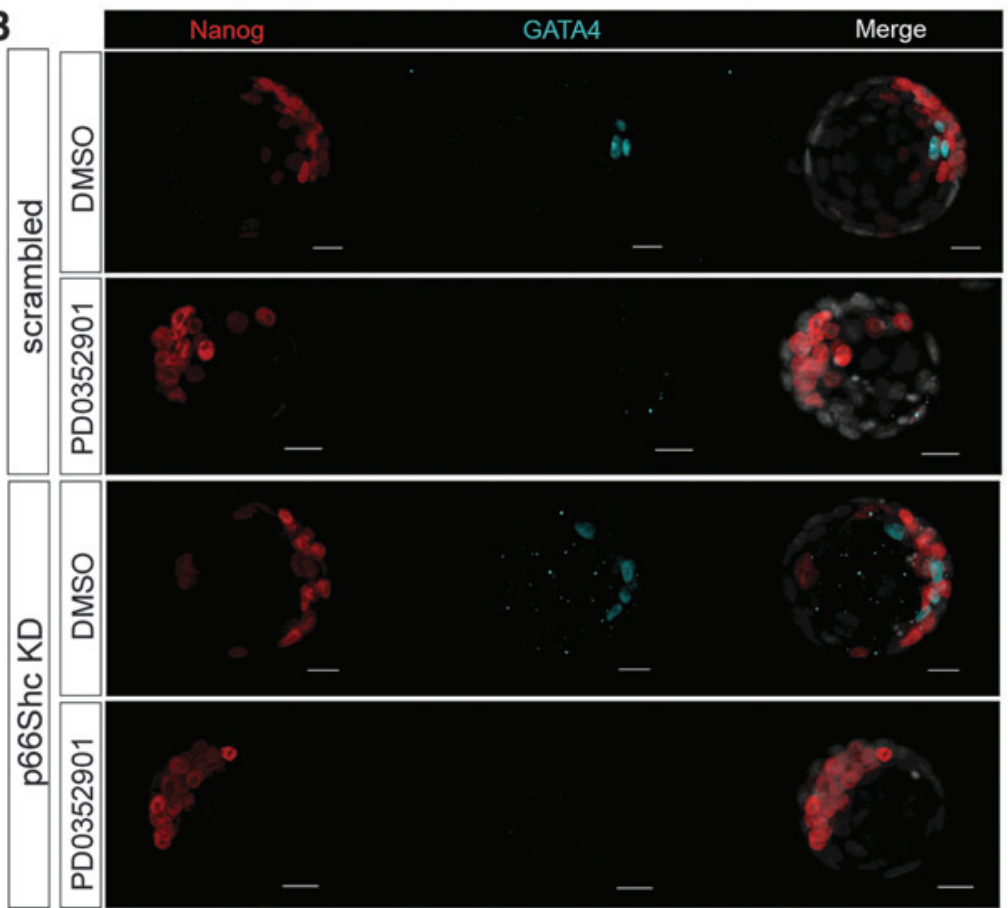

FIG. 7. p66Shc knockdown blastocysts displayed altered ERK1/2 activity. (A) p66Shc knockdown blastocysts had a significant increase in cells positive for DUSP4, an ERK1/2 transcriptional target downstream of FGF/ MAPK signaling, compared with controls $(n=13$ blastocysts, mean \pm SEM, $* P<0.05)$. (B) When treated with $1 \mu \mathrm{M}$ PD0325901, all cells of the inner cell mass of p66Shc knockdown blastocysts were NANOG positive $(n=17$ blastocysts per group). Scale bars are $20 \mu \mathrm{m}$. ERK, extracellular signal-regulated kinase; FGF, fibroblast growth factor; MAPK, mitogen-activated protein kinase. 
immunofluorescence and confocal microscopy (Supplementary Fig. S5A). To overcome the challenge of reliably detecting ERK1/2 activation, we proceeded to define DUSP4 localization, a transcriptional target of FGF4MAPK signaling specific to ICM cells committing to the PE fate [25]. Accordingly, we observed DUSP4 colocalization with GATA4 in blastocysts (Fig. 7A). We did not observe a significant change in DUSP4-positive cells or Dusp4 transcript abundance in embryos $72 \mathrm{~h}$ post siRNA injection (Supplementary Fig. S5B). However, p66Shc knockdown blastocysts at $96 \mathrm{~h}$ post siRNA injection contained a significantly higher percentage of DUSP4-positive cells compared with scrambled controls, suggesting that these blastocysts have more cells with active ERK1/2 compared with controls (Fig. 7A). The increased proportion of PE cells in the ICM of p66Shc knockdown blastocysts was reversed by ERK1/2 inhibition, as treatment for $24 \mathrm{~h}$ with $1 \mu \mathrm{M}$ PD0325901 resulted in all inner cells of p66Shc knockdown blastocysts to become NANOG positive at $96 \mathrm{~h}$ post siRNA injection (Fig. 7B). Altogether, our results support that decreased expression of p66Shc is associated with an increase in the number of cells in the blastocyst with active ERK1/2, and that the increase in GATA4-positive cells in the ICM is reversible by MEK1/2 inhibition.

\section{Discussion}

In this study, we show a novel role for p66Shc during mouse preimplantation development, demonstrating that p66Shc is mechanistically linked to the expression dynamics of blastocyst lineage-associated transcription factors. Our results demonstrate that knockdown of p66Shc during preimplantation embryo development affects the abundance and restriction of OCT3/4 and the number of cells expressing markers of the PE (GATA4, SOX17) in the ICM. Knockdown of p66Shc in mouse embryos leads to blastocysts with more GATA4-positive cells than NANOG-positive cells in the ICM compared with controls. This imbalance is partially resolved by the late blastocyst stage, but PE cells appear to sort to the PE layer earlier in knockdown blastocysts compared with controls. When plated in vitro, p66Shc knockdown blastocysts have a decreased ability to generate ICM-derived outgrowths compared with controls. Furthermore, decreased p66Shc expression leads to an increased number of cells expressing DUSP4, and the increased proportion of GATA4expressing cells is rescued by ERK1/2 inhibition. Our study also suggests a possible novel role for p66Shc in regulating the cell signaling that results in the commitment to extraembryonic endoderm.

Regulation of cell fate-associated transcription factor expression is a novel proposed function for p66Shc, as the gene has thus far been shown to negatively regulate embryo development by promoting apoptosis and senescence (embryo arrest). However, many of these effects were stimulated by stressful environmental conditions, including treatment with $\mathrm{H}_{2} \mathrm{O}_{2}$ [16] and arsenic [17]. In these cases, p66Shc may act to ensure that embryos unable to adapt to stress do not continue to develop by inducing mitochondrial ROS to promote apoptosis or embryo arrest. In contrast to studies with bovine embryos, we did not observe a significant increase in the number of embryos developing into blastocysts after p66Shc knockdown compared with con- trols, suggesting that knockdown of p66Shc in our experimental model did not improve basal levels of blastocyst development [15]. Interestingly, our results also suggest that p66Shc knockdown blastocysts may be more developmentally advanced than controls, which suggests that they are following the developmental timeline of embryos developing in vivo. It is well known that late blastocyst development is delayed in embryos cultured in vitro relative to their in vivo counterparts [21]. However, culture does not appear to significantly alter the timing of lineage-specific marker patterning, as in vivo derived blastocysts also show initial OCT3/4 expression in the TE until the late blastocyst stage [21]. This apparent developmental advancement in p66Shc knockdown embryos may be due to enhanced stress resistance to the culture environment conferred by reduced p66Shc expression.

We were unable to detect differences in MAPK signaling between p66Shc knockdown and control embryos at $72 \mathrm{~h}$ post siRNA injection, when p66Shc protein expression is still significantly decreased by RNAi. Instead, we observed phenotypic differences at the blastocyst stage, when the knockdown effect is no longer present at the transcriptional level. RNA interference using siRNAs is a limitation of our study as knockdown effects did not persist at the transcript level to the blastocyst stage $[14,15]$. However, due to protein turnover and stability, the timing of maximal phenotypic effects does not necessarily correspond to maximal knockdown effects. Several studies using siRNA to knockdown p66Shc in preimplantation embryos also observe restoration of p66Shc transcript levels to wild-type levels at the blastocyst stage, but the transient knockdown of p66Shc during preimplantation development significantly affected the ability of blastocysts to develop under basal and stressinducing culture conditions [15-17]. Our results suggest that transient knockdown of p66Shc up to $72 \mathrm{~h}$ of development has an effect on cell-lineage transcription factor expression at the blastocyst stage. This could be through affecting earlier events in PE specification such as differences in FGF4/FGFR2 signaling that first occurs in the early ICM [26], or through regulating the levels of OCT3/4 in morulae that in turn would affect FGF4 levels [21,27]. Additionally, there may be effects of p66Shc on other signaling pathways that have not yet been identified but are independently required for the onset of Gata6 expression [6].

Interestingly, EPI survival was reduced in vitro in p66Shc knockdown blastocysts as significantly fewer blastocysts produced ICM outgrowths compared with scrambled controls. We did not test whether p66Shc knockdown enhanced the survival of the PE or the TE in vitro. We speculate that the derivation of extraembryonic endoderm and/or trophoblast stem cells may be enhanced in p66Shc knockdown blastocysts due to the increased number of cells positive for GATA4 (PE) and CDX2 (TE) observed at the blastocyst stage. We did not quantify this as the conditions we used (knockout serum replacement [KOSR] and leukemia inhibitory factor [LIF]) predominately support EPI survival [28]. The importance of p66Shc to pre- and periimplantation survival is still not well understood. P66Shc KO mice survive embryonic development, however, it is unclear whether mating between homozygous null mice was tested [13]. P66Shc mRNA and/or protein stored in the oocyte of female heterozygous KO mice may produce a maternal rescue of a preimplantation phenotype. However, when exposed to natural environmental 
conditions (eg, not a controlled laboratory environment), p66Shc KO mice have reduced fertility with repeated breeding cycles [29]. To this end, our future experiments are being directed toward producing maternal and embryonic genetic KO models of p66Shc in preimplantation embryos and the production of these lines will determine the extent of p66Shc regulation of OCT3/4 expression during preimplantation development, its requirement for establishing cell fate in the ICM, and the consequences of its loss on fetal and postpartum development.

Knockdown of p66Shc during mouse preimplantation development resulted in morphologically normal blastocysts. However, we detected an increased total cell number in p66Shc knockdown blastocysts compared with controls, and attribute this increase to altered proliferation in the TE. p66Shc has not been extensively linked with cell cycle regulation in other studies, but it could indirectly affect cell proliferation through regulating epidermal growth factor receptor (EGFR) signaling activity [11]. The time frame for altered proliferation is short, as knockdown embryos $72 \mathrm{~h}$ post siRNA injection contained fewer total cells, and knockdown embryos $120 \mathrm{~h}$ postinjection did not significantly differ in cell number than controls.

After investigating the transcript levels of cell cycle markers, we observed a significant decrease in $R b 1$ transcript abundance in p66Shc knockdown blastocysts. Rb1 restricts the G1/S checkpoint of the cell cycle by binding and repressing E2F transcription factors [30]. Regulation of RB1 function is typically by phosphorylation and not by transcriptional regulation. However, RB1 is involved in promoting self-renewal and repressing pluripotency networks, including OCT3/4 and SOX2 expression, independent of the cell cycle [31,32]. The increase in OCT3/4 expression in p66Shc knockdown blastocysts observed in our study could be functionally linked to the decreased $R b l$ transcript abundance. The effects of p66Shc knockdown to other key cell cycle genes (Ccndl, Ccnel) would be useful and would provide insight into the role of p66Shc in cell cycle regulation in the mouse blastocyst. Other studies with EPI/PE imbalance phenotypes also have altered proliferation, suggesting that the cell cycle may be linked to cell fate specification in the mouse blastocyst $[33,34]$.

We observed significantly higher OCT3/4 levels, faster restriction of OCT3/4 to the inner cells of the blastocyst with p66Shc knockdown, and a significantly increased proportion of cells expressing PE markers (GATA4 and SOX17) in p66Shc knockdown blastocysts. This increase in OCT3/4 levels could directly promote PE differentiation in the ICM of p66Shc knockdown blastocysts. Palmieri et al. observed higher OCT3/4 fluorescence intensity in the forming PE relative to the rest of the ICM, which led to speculation that elevation of OCT3/4 levels may be a prerequisite to PE differentiation [22]. OCT3/4 is required to promote PE fate in the mouse blastocyst by regulating the expression of FGF4 and PE markers [27,35,36]. Additionally, a less than twofold overexpression of OCT3/4 in embryonic stem cells is sufficient to promote endoderm differentiation [23]. Our work suggests that PE marker expression may be a direct consequence of OCT3/4 elevation in the ICM. This raises the possibility that p66Shc may regulate $\mathrm{PE}$ marker expression by directly or indirectly regulating OCT3/4 levels in the ICM.

Our results suggest that the effects of p66Shc on the expression of SOX17 and GATA4 are mediated in part through ERK1/2 signaling. However, we did not directly confirm that these effects were downstream or upstream of FGF4 and cannot conclude that p66Shc regulates this specific pathway. No other signaling pathway aside from FGF4/ MAPK has been identified as being critical for promoting PE marker expression and PE fate, and thus we speculate that in the blastocyst p66Shc is acting as a canonical adaptor protein to negatively regulate ERK $1 / 2$ activity downstream of FGF4/RTK activation.

It is possible that p66Shc could be acting through another RTK, such as EGFR, and ERK1/2 activity is regulated through pathway crosstalk. For example, p66Shc overexpression is associated with increased $\beta$-catenin activity in mouse embryonic stem cells undergoing directed neural differentiation, which may be regulated through p66Shc-mediated generation of ROS [37]. Although its interactions with the EGFR are the most well studied, there is evidence that Shc proteins are recruited to and phosphorylated by FGFR1, 3, and 4, and indirectly associate with FGFR2 in vivo [38]. It is also possible that FGFR1 versus FGFR2 expression is altered downstream of p66Shc knockdown, which would affect the EPI/PE balance in the ICM [25]. However, our results also suggest an intriguing possibility that p66Shc may regulate this pathway by regulating OCT3/4 levels. P66Shc may ensure that OCT3/4 levels do not reach a threshold that would promote PE marker expression and subsequent differentiation. Our work outlines an additional novel role of p66Shc in mouse blastocyst development associated with the onset and relative abundance of lineageassociated transcription factors.

\section{Acknowledgments}

The authors thank Dr. John DiGuglielmo for providing the micromanipulation equipment, Dr. Lina Dagnino for providing BrdU reagents, and Dr. Cheryle Seguin and Courtney Brooks (The University of Western Ontario, Canada) for providing mouse embryonic stem cells. Confocal microscopy was carried out at the Integrated Microscopy Laboratory at the Biotron Experimental Climate Change Research Center (The University of Western Ontario, Canada). This work is supported by a Canadian Institutes of Health Research operating grant to A.J.W. and D.H.B. (MOP 130396). N.A.E. is supported by a Natural Sciences and Engineering Research Council Doctoral Canada Graduate Scholarship.

\section{Author Disclosure Statement}

No competing financial interests exist.

\section{References}

1. Nishioka N, K Inoue, K Adachi, H Kiyonari, M Ota, A Ralston, N Yabuta, S Hirahara, RO Stephenson, et al. (2009). The Hippo signaling pathway components Lats and Yap pattern Tead4 activity to distinguish mouse trophectoderm from inner cell mass. Dev Cell 16:398-410.

2. Cockburn K, S Biechele, J Garner and J Rossant. (2013). The Hippo pathway member Nf2 is required for inner cell mass specification. Curr Biol 23:1195-1201.

3. Hirate Y, S Hirahara, K Inoue, A Suzuki, VB Alarcon, K Akimoto, T Hirai, T Hara, M Adachi, et al. (2013). Polarity-dependent distribution of angiomotin localizes 
Hippo signaling in preimplantation embryos. Curr Biol 23: 1181-1194.

4. Krawchuk D, N Honma-Yamanaka, S Anani and Y Yamanaka. (2013). FGF4 is a limiting factor controlling the proportions of primitive endoderm and epiblast in the ICM of the mouse blastocyst. Dev Biol 384:65-71.

5. Yamanaka Y, F Lanner and J Rossant. (2010). FGF signaldependent segregation of primitive endoderm and epiblast in the mouse blastocyst. Development 137:715-724.

6. Frankenberg S, F Gerbe, S Bessonnard, C Belville, P Pouchin, O Bardot and C Chazaud. (2011). Primitive endoderm differentiates via a three-step mechanism involving Nanog and RTK signaling. Dev Cell 21:1005-1013.

7. Nichols J, J Silva, M Roode and A Smith. (2009). Suppression of Erk signalling promotes ground state pluripotency in the mouse embryo. Development 136:32153222.

8. Lorthongpanich C, DM Messerschmidt, SW Chan, W Hong, BB Knowles and D Solter. (2013). Temporal reduction of LATS kinases in the early preimplantation embryo prevents ICM lineage differentiation. Genes Dev 27: 1441-1446.

9. Chazaud C, Y Yamanaka, T Pawson and J Rossant. (2006). Early lineage segregation between epiblast and primitive endoderm in mouse blastocysts through the Grb2-MAPK pathway. Dev Cell 10:615-624.

10. Grabarek JB, K Zyzyńska, N Saiz, A Piliszek, S Frankenberg, J Nichols, AK Hadjantonakis and B Plusa. (2012). Differential plasticity of epiblast and primitive endoderm precursors within the ICM of the early mouse embryo. Development 139:129-139.

11. Migliaccio E, S Mele, AE Salcini, G Pelicci, KM Lai, G Superti-Furga, T Pawson, PP Di Fiore, L Lanfrancone and PG Pelicci. (1997). Opposite effects of the p52shc/p46shc and p66shc splicing isoforms on the EGF receptor-MAP kinase-fos signalling pathway. EMBO J 16:706-716.

12. Okada S, AW Kao, BP Ceresa, P Blaikie, B Margolis and JE Pessin. (1997). The 66-kDa Shc isoform Is a negative regulator of the epidermal growth factor-stimulated mitogen-activated protein kinase pathway. J Biol Chem 272:28042-28049.

13. Migliaccio E, M Giorgio, S Mele, G Pelicci, P Reboldi, PP Pandolfi, L Lanfrancone and PG Pelicci. (1999). The p66shc adaptor protein controls oxidative stress response and life span in mammals. Nature 402:309-313.

14. Edwards NA, AJ Watson and DH Betts. (2016). P66Shc, a key regulator of metabolism and mitochondrial ROS production, is dysregulated by mouse embryo culture. Mol Hum Reprod 22:634-647.

15. Betts DH, NT Bain and P Madan. (2014). The p66(Shc) adaptor protein controls oxidative stress response in early bovine embryos. PLoS One 9:e86978.

16. Favetta LA, P Madan, GF Mastromonaco, EJSt St John, WA King and DH Betts. (2007). The oxidative stress adaptor p66Shc is required for permanent embryo arrest in vitro. BMC Dev Biol 7:132.

17. Ren K, X Li, J Yan, G Huang, S Zhou, B Yang, X Ma and C Lu. (2014). Knockdown of p66Shc by siRNA injection rescues arsenite-induced developmental retardation in mouse preimplantation embryos. Reprod Toxicol 43:8-18.

18. Calder MD, NA Edwards, DH Betts, and AJ Watson. (2017). Treatment with AICAR inhibits blastocyst devel- opment, trophectoderm differentiation and tight junction formation and function in mice. Mol Hum Reprod 23:771785.

19. Lou X, M Kang, P Xenopoulos, S Muñoz-Descalzo and AK Hadjantonakis. (2014). A rapid and efficient 2D/3D nuclear segmentation method for analysis of early mouse embryo and stem cell image data. Stem Cell Rep 2:382-397.

20. Xie Y, EE Puscheck and DA Rappolee. (2006). Effects of SAPK/JNK inhibitors on preimplantation mouse embryo development are influenced greatly by the amount of stress induced by the media. Mol Hum Reprod 12:217224.

21. Dietrich JE and T Hiiragi. (2007). Stochastic patterning in the mouse pre-implantation embryo. Development 134: 4219-4231.

22. Palmieri SL, W Peter, H Hess and HR Schöler. (1994). Oct4 transcription factor is differentially expressed in the mouse embryo during establishment of the first two extraembryonic cell lineages involved in implantation. Dev Biol 166:259-267.

23. Niwa H, J Miyazaki and AG Smith. (2000). Quantitative expression of Oct-3/4 defines differentiation, dedifferentiation or self-renewal of ES cells. Nat Genet 24:372-376.

24. Plusa B, A Piliszek, S Frankenberg, J Artus and AK Hadjantonakis. (2008). Distinct sequential cell behaviours direct primitive endoderm formation in the mouse blastocyst. Development 135:3081-3091.

25. Kang M, V Garg and AK Hadjantonakis. (2017). Lineage establishment and progression within the inner cell mass of the mouse blastocyst requires FGFR1 and FGFR2. Dev Cell 41:496-510.e5.

26. Guo G, M Huss, GQ Tong, C Wang, L Li Sun, ND Clarke and P Robson. (2010). Resolution of cell fate decisions revealed by single-cell gene expression analysis from zygote to blastocyst. Dev Cell 18:675-685.

27. Nichols J, B Zevnik, K Anastassiadis, H Niwa, D KleweNebenius, I Chambers, H Schöler and A Smith. (1998). Formation of pluripotent stem cells in the mammalian embryo depends on the POU transcription factor Oct4. Cell 95:379-391.

28. Behringer R, M Gertsenstein, K Vintersten Nagy and A Nagy. (2014). Manipulating the Mouse Embryo: A Laboratory Manual, 4th edn. Cold Spring Harbor Laboratory Press, Cold Spring Harbor, NY.

29. Giorgio M, A Berry, I Berniakovich, I Poletaeva, M Trinei, M Stendardo, K Hagopian, JJ Ramsey, G Cortopassi, et al. (2012). The p66Shc knocked out mice are short lived under natural condition. Aging Cell 11:162-168.

30. Weinberg RA. (1995). The retinoblastoma protein and cell cycle control. Cell 81:323-330.

31. Conklin JF, J Baker and J Sage. (2012). The RB family is required for the self-renewal and survival of human embryonic stem cells. Nat Commun 3:1244.

32. Kareta MS, LL Gorges, S Hafeez, BA Benayoun, S Marro, AF Zmoos, MJ Cecchini, D Spacek, LF Batista, et al. (2015). Inhibition of pluripotency networks by the Rb tumor suppressor restricts reprogramming and tumorigenesis. Cell Stem Cell 16:39-50.

33. Azami T, T Waku, K Matsumoto, H Jeon, M Muratani, A Kawashima, J Yanagisawa, I Manabe, R Nagai, et al. (2017). Klf5 maintains the balance of primitive endoderm versus epiblast specification during mouse embryonic 
development by suppression of Fgf4. Development 144: 3706-3718.

34. Chen YH and J Yu. (2012). Ectopic expression of Fgf3 leads to aberrant lineage segregation in the mouse parthenote preimplantation embryos. Dev Dyn 241:1651-1664.

35. Le Bin GC, S Muñoz-Descalzo, A Kurowski, H Leitch, X Lou, W Mansfield, C Etienne-Dumeau, N Grabole, C Mulas, et al. (2014). Oct4 is required for lineage priming in the developing inner cell mass of the mouse blastocyst. Development 141:1001-1010.

36. Frum T, MA Halbisen, C Wang, H Amiri, P Robson and A Ralston. (2013). Oct4 cell-autonomously promotes primitive endoderm development in the mouse blastocyst. Dev Cell 25:610-622.

37. Papadimou E, A Moiana, D Goffredo, P Koch, S Bertuzzi, O Brüstle, E Cattaneo and L Conti. (2009). p66(ShcA) adaptor molecule accelerates ES cell neural induction. Mol Cell Neurosci 41:74-84.

38. Schüller AC, Z Ahmed, JA Levitt, KM Suen, K Suhling and JE Ladbury. (2008). Indirect recruitment of the sig- nalling adaptor Shc to the fibroblast growth factor receptor 2 (FGFR2). Biochem J 416:189-199.

Address correspondence to:

Dr. Dean H. Betts

Department of Physiology and Pharmacology Schulich School of Medicine \& Dentistry The University of Western Ontario Dental Sciences Building Room 2022 1151 Richmond Street N6A $3 K 7$ London Canada

E-mail: dean.betts@schulich.uwo.ca

Received for publication June 18, 2018

Accepted after revision August 8, 2018

Prepublished on Liebert Instant Online August 9, 2018 